\title{
Treating leptomeningeal metastases from primary hepatic neuroendocrine carcinoma with combined radiotherapy and immunotherapy: a case report
}

\author{
Wei-Jun Wang ${ }^{1}$, Kuan-Der Lee ${ }^{2}$, Long-Sheng Lu ${ }^{1,3}$, Lai-Lei Ting ${ }^{1}$, Jeng-Fong Chiou, ${ }^{1,4}$ \\ ${ }^{1}$ Department of Radiation Oncology, Taipei Medical University Hospital, Taipei, Taiwan; ${ }^{2}$ Division of Hematology and Oncology, Department \\ of Internal Medicine, Taipei Medical University Hospital, Taipei, Taiwan; ${ }^{3}$ Graduate Institute of Biomedical Materials and Tissue Engineering, \\ College of Biomedical Engineering, Taipei Medical University, Taipei, Taiwan; ${ }^{4}$ Department of Radiology, School of Medicine, College of \\ Medicine, Taipei Medical University, Taipei, Taiwan \\ Correspondence to: Lai-Lei Ting. Department of Radiation Oncology, Taipei Medical University Hospital, No.252, Wu-Hsing St., Taipei 11031, \\ Taiwan. Email: 1lt1957@gmail.com.
}

\begin{abstract}
Radiotherapy has been applied for decades as a treatment modality in the management of various type of cancer, either in curative or palliative intent. Recently, evidence accumulated that immune checkpoint inhibitors may have noninferior treatment effects when compared with conventional systemic therapy. Because ionizing radiation induces local inflammatory effects, it is postulated that the response to checkpoint blockade immunotherapy may be improved if it is administered in conjunction with radiotherapy in treating malignancy. This interesting phenomenon has been observed in many types of cancers. Here we reported a 62-year-old woman who was diagnosed as having primary hepatic neuroendocrine carcinoma (NEC) with diffuse metastases including leptomeningeal metastases (LM) accompanied with deteriorated conditions. She underwent whole-brain radiotherapy followed by immunotherapy. She adequately tolerated the treatment, and her symptoms were alleviated rapidly with improved consciousness. Magnetic resonance imaging (MRI) conducted 1 month after the treatment revealed significant disappearance of the LM, but tumors elsewhere progressed. The patient's overall survival time was 27 months, and she had survived for 3 months from the time of diagnosis of LM. Reported clinical trials and retrospective studies combining immunotherapy and radiotherapy demonstrated a therapeutic potency due to the augmentation of the immune-mediated responses, and more studies are still on going. Treating LM with radiation-assisted immunotherapy is a feasible treatment option with potential clinical benefit, and further study of its utilities and risks is warranted.
\end{abstract}

Keywords: Case report; immunotherapy; meningeal carcinomatosis; neuroendocrine carcinoma (NEC); radiotherapy

Received: 11 October 2019; Accepted: 21 January 2021; Published: 30 March 2021.

doi: $10.21037 /$ tro-19-103

View this article at: http://dx.doi.org/10.21037/tro-19-103

\section{Introduction}

In the past decade, checkpoint inhibitors have engendered considerable changes in several immunotherapy strategies. Despite the rapid advancement in checkpoint inhibitor immunotherapy, the utility of such immunotherapy in the treatment of neuroendocrine tumors (NETs) is relatively unknown, and treatment options for progressive disease are limited. Combination of immune checkpoint inhibitor and radiotherapy can enhance the treatment effects, which has been documented in melanoma, lung cancer, and other metastatic diseases. Herein, we report the case of a 62-yearold woman with primary hepatic neuroendocrine carcinoma 

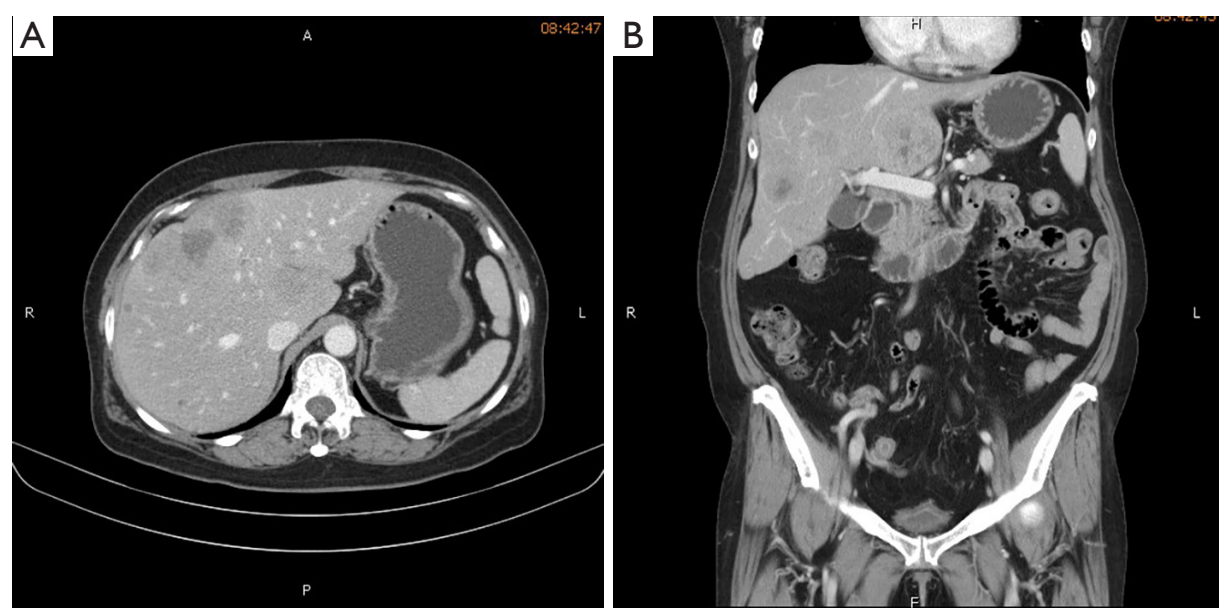

Figure 1 Contrast-enhanced CT scan of the abdomen. (A) Axial and (B) coronal views revealing multiple tumors in both liver lobes. CT, computed tomography.

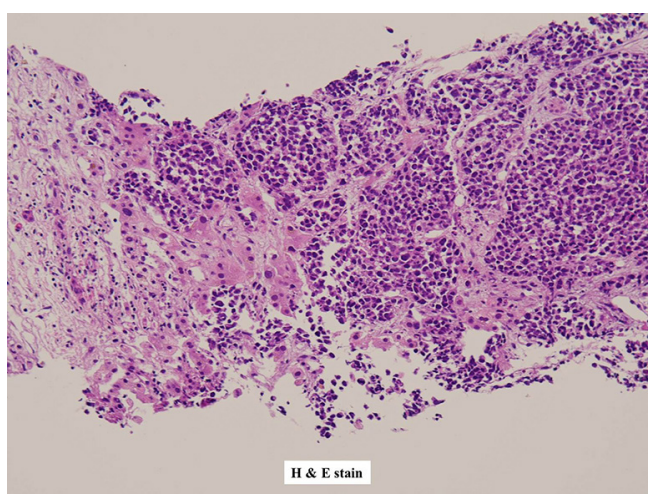

Figure 2 Histological and immunohistochemical staining, 200x.

(NEC) who developed leptomeningeal metastases (LM) following combined treatment with two cycles of nivolumab $200 \mathrm{mg}$ per dose and whole-brain radiotherapy in 3 weeks with complete response. We present the following case in accordance with the CARE reporting checklist. (available at http://dx.doi.org/10.21037/tro-19-103).

\section{Case presentation}

A 62-year-old Taiwanese woman with primary hepatic NEC had received first-line and salvage chemotherapy with partial response for 2 years, but the disease progressed later. Before she denied any medical or family history. She visited our hospital to seek a second opinion. An enhanced computed tomography scan revealed multiple hepatic tumors (Figure 1); these tumors were subsequently confirmed pathologically through a liver biopsy to be poorly differentiated carcinoma with neuroendocrine differentiation (Figure 2). Microscopically, we observed solid sheets infiltrating the liver parenchyma that were composed of pleomorphic tumor cells with hyperchromatic nuclei, salt-and-pepper chromatin, indistinct nucleoli, light eosinophilic cytoplasm, frequent mitosis, and tumor necrosis. Immunohistochemically, the tumor cells were positive for cytokeratin, CD117, CD56, and chromogranin; negative for synaptophysin; and weakly positive for PD-L1 expression. A few days after the diagnosis, she developed severe dizziness followed by reduced consciousness and epileptic seizures. Conventional biochemical examinations including routine blood, urine, liver, and kidney function tests revealed normal results. Enhanced magnetic resonance imaging (MRI) reported disseminated metastatic lesions in bilateral cerebral hemispheres and cerebrospinal fluid (CSF) spaces, resulting in diffuse pial matter enhancement in the supra-and infratentorial spaces. (Figure 3). At that time, the patient and her family refused aggressive intervention such as CSF analysis, intrathecal chemotherapy or craniospinal irradiation. The patient underwent whole-brain threedimensional conformal radiotherapy with 6-MV X-ray beams; the radiotherapy process involved a total radiation dose of 30 Gy administered in 15 fractions (Figures 4,5) along with two cycles of nivolumab administered at $200 \mathrm{mg}$ per dose in 3 weeks. During the treatment, neutropenia, thrombopenia, and seizures were observed but were resolved with medication. The patient tolerated the treatment well, and her symptoms were rapidly alleviated. 

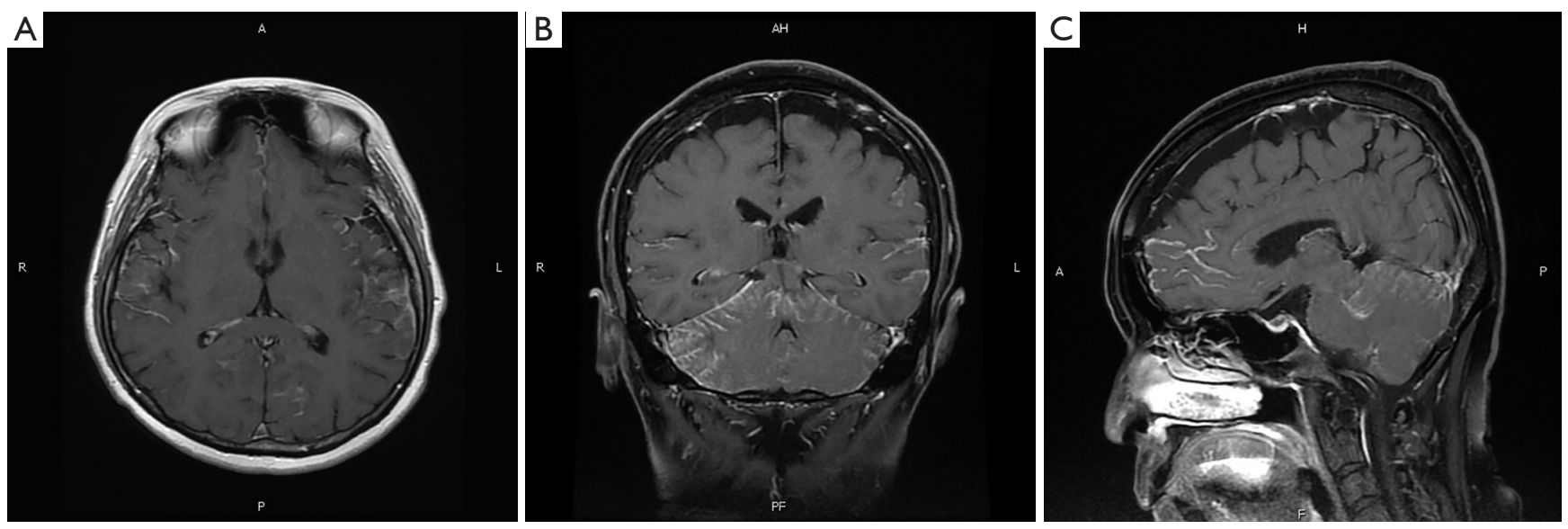

Figure 3 Contrast-enhanced brain MRI. (A) Axial T1, (B) coronal T1, and (C) sagital T1 MRI revealing disseminated metastatic lesions in the bilateral cerebral hemispheres and CSF spaces. MRI, magnetic resonance imaging; CSF, cerebrospinal fluid.
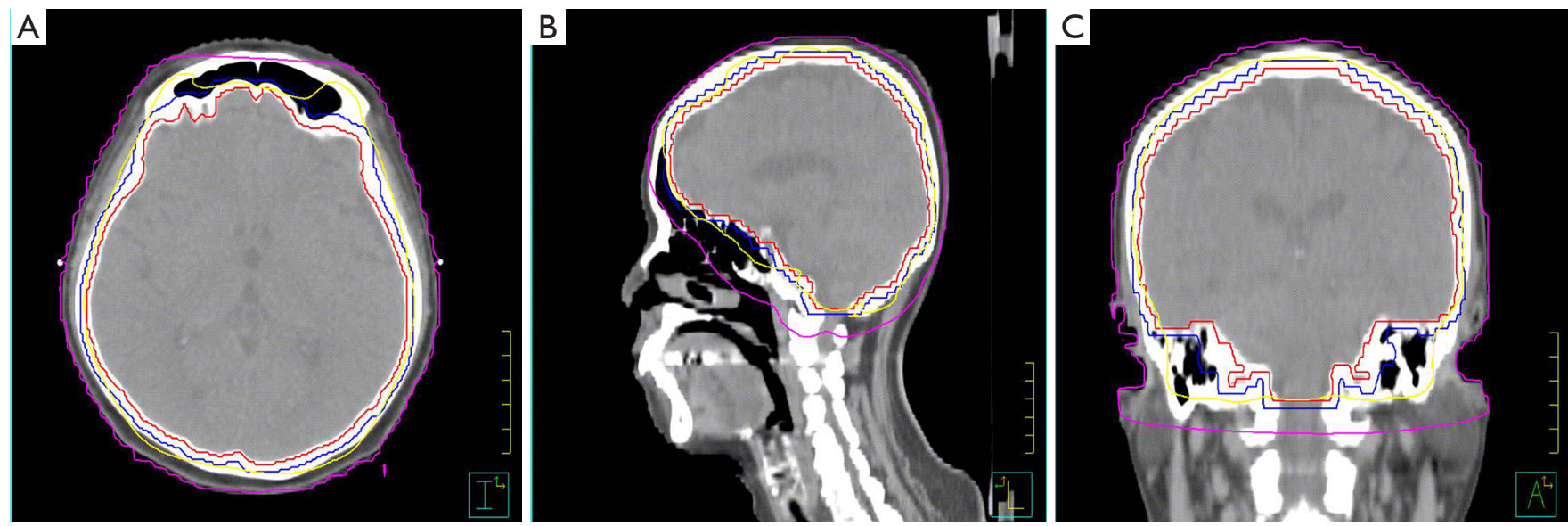

Figure 4 Dose distributions of the treatment plan in (A) axial, (B) sagittal, and (C) coronal planes. The patient underwent whole-brain three-dimensional conformal radiotherapy with 6-MV X-ray beams; the radiotherapy involved a total radiation dose of 30 Gy administered in 15 fractions. The CTV (red), PTV (blue), and isodose lines corresponding to $100 \%$ (yellow) and 50\% (purple) of the prescribed dose are presented. CTV, clinical target volume; PTV, planning target volume.

An MRI process conducted 1 month after the treatment revealed disappearance of the LM (Figure 6), but the primary hepatic tumors had progressed (Figure 7). After the patient received two additional cycles of nivolumab, her disease progressed with an increase in hepatic nodules (Figure 8). She died because of septic shock but without signs of hepatic failure. The patient's overall survival time was 27 months, and she had survived for 3 months from the time of diagnosis of LM (Figure 9). All procedures performed in studies involving human participants were in accordance with the ethical standards of the institutional and/or national research committee(s) and with the Helsinki Declaration (as revised in 2013). The consent was waived for this study by the IRB committee at the Taipei Medical University Hospital since the patient died. The study was approved by Taipei Medical University-Joint Institutional Review Board (N201710026).

\section{Discussion}

NETs are rare and can originate in neuroendocrine cells throughout the body. According to the Surveillance, 


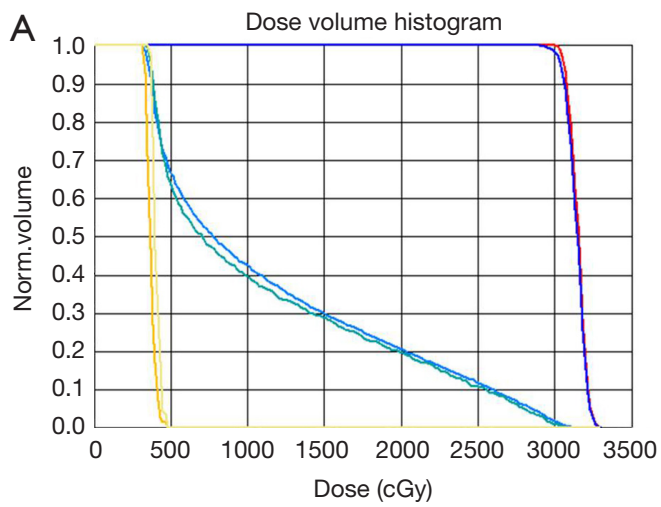

\begin{tabular}{|c|c|c|c|c|c|c|}
\hline \multicolumn{7}{|c|}{ ROI Statistics } \\
\hline & $\begin{array}{l}\text { Line } \\
\text { Type }\end{array}$ & $\mathrm{ROI}$ & $\begin{array}{l}\text { Trial or } \\
\text { Record }\end{array}$ & Min. & Max. & Mean \\
\hline$\vee$ & - & CTV_200*15 & Whole brain & 2907.7 & 3298.1 & 3149.7 \\
\hline$\checkmark$ & - & L't eye & Whole brain & 299.9 & 3113.8 & 1143.5 \\
\hline$\diamond$ & - & L't lens & Whole brain & 315.2 & 471.3 & 367.4 \\
\hline$\diamond$ & - & PTV +3 mm & Whole brain & 2304.6 & 3298.1 & 3140.1 \\
\hline$\diamond$ & - & R't eye & Whole brain & 301.8 & 3076.9 & 1102.6 \\
\hline$\diamond$ & - & R't lens & Whole brain & 360.8 & 465.4 & 401.4 \\
\hline
\end{tabular}

Figure 5 Dose-volume histogram for the (A) tumor and (B) critical organs.
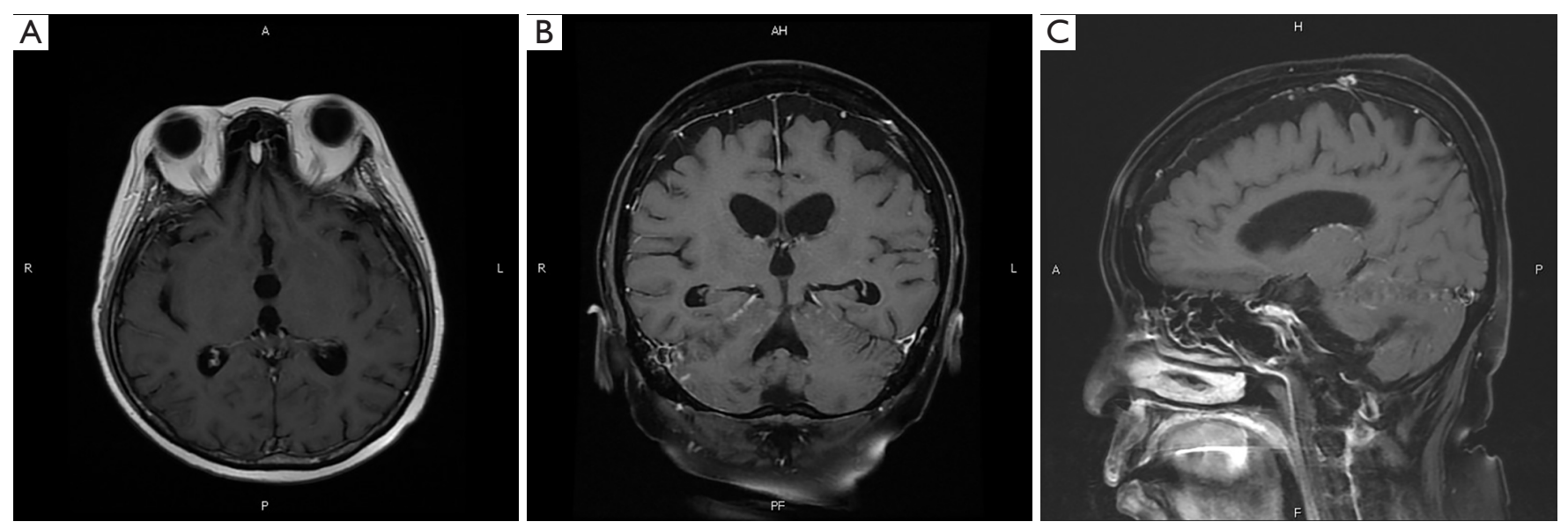

Figure 6 Contrast-enhanced brain MRI 1 month after radiotherapy. (A) Axial T1, (B) coronal T1, and (C) sagital T1 MRI results revealing interval improvement with complete disappearance of the leptomeningeal metastatic nodular tumors. MRI, magnetic resonance imaging.
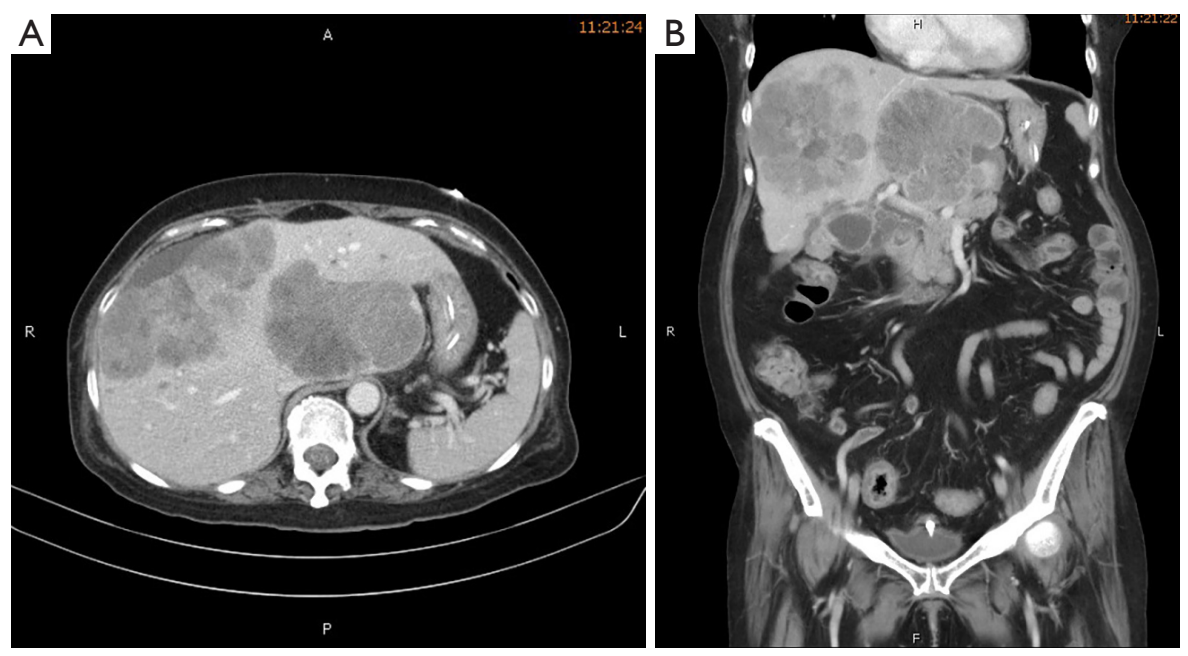

Figure 7 Contrast-enhanced CT scan of the abdomen 1 month after completion of brain irradiation. (A) Axial and (B) coronal views revealing progression of hepatic tumors. 

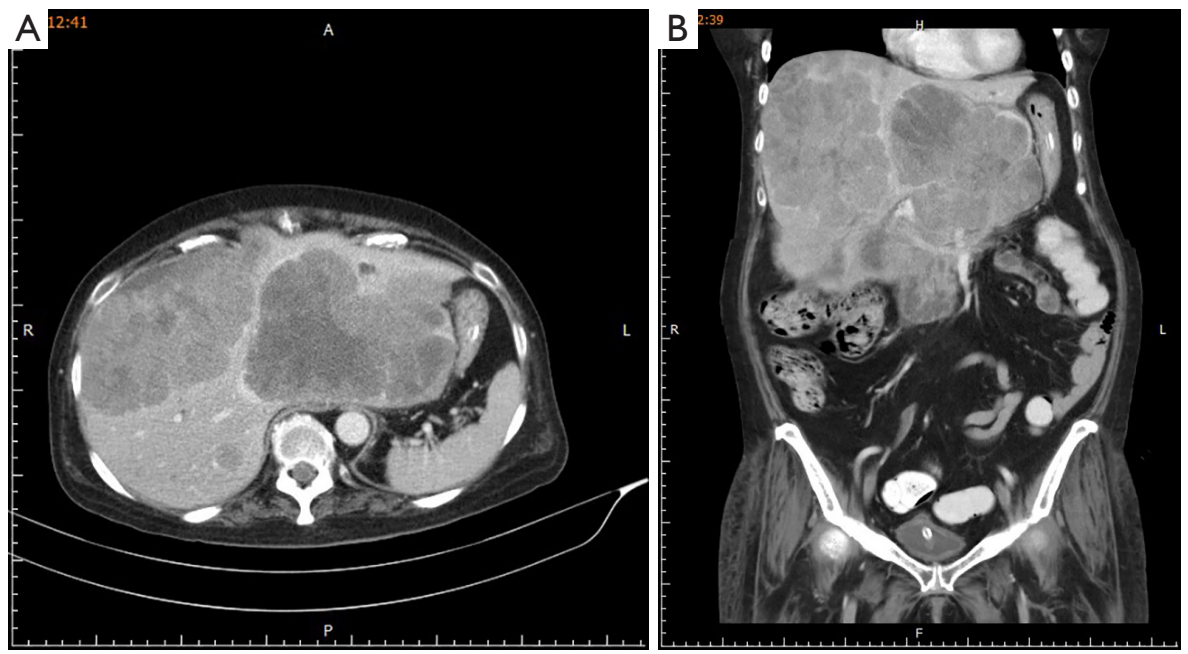

Figure 8 Contrast-enhanced CT scan of the abdomen 2 months after completion of brain irradiation. (A) Axial and (B) coronal views revealing disease progression with considerable enlargement of lesions as well as an increased number in the liver.

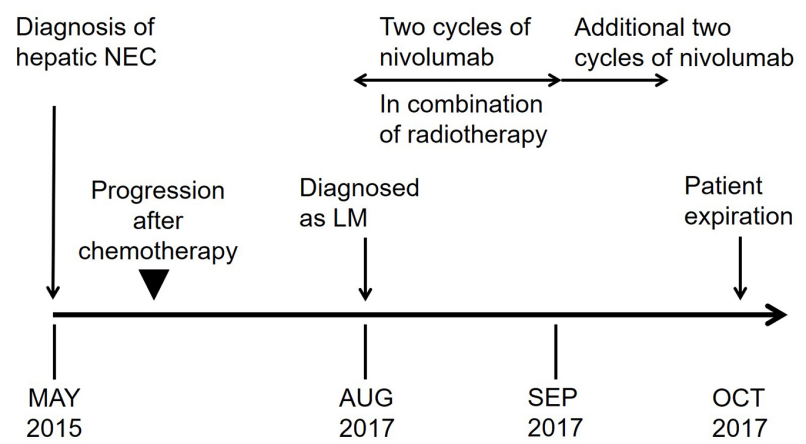

Figure 9 Disease course and treatment timeline of the patient.

Epidemiology, and End Result (SEER) program database, in the United States, the annual incidence of NETs originating in the lung is approximately 1.49 per 100,000 , that of NETs originating at gastroenteropancreatic sites is 3.56 per 100,000, and that of NETs originating at unknown primary sites is 0.84 per 100,000 . In 2017, the World Health Organization defined three NET categories based on histology: well-differentiated, low-grade NETs (G1); intermediate-grade NETs (G2); and poorly differentiated, high grade NECs. The tumors are staged according to the American Joint Committee on Cancer tumor-nodemetastasis system but vary for different primary locations. However, no staging system has been established specifically for primary hepatic NETs because of their rarity. Poorly differentiated NEC is often associated with an aggressive clinical course. A study analyzing the SEER database indicated that the median survival times of patients with lung NEC, gastrointestinal NEC, and unknown primary NEC were 7.6, 7.5, and 2.5 months, respectively (1). Another study on patients with NEC revealed that metastases were found in $20.8 \%$ of the patients at presentation and in an additional $38 \%$ of the patients after the initial diagnosis (2). Because most NEC tumors are locally advanced or metastatic at presentation, surgery alone is rarely curative; combined modalities are typically recommended to address the disease's rapid progression. Radiotherapy is safe and effective for achieving local control, particularly for surgically unresectable tumors. A retrospective study examined 36 patients with pancreatic NETs who received radiotherapy for primary tumors or symptomatic metastases (3); the overall response rate to radiotherapy was 39\% (13\% complete response, $26 \%$ partial response, $56 \%$ stable disease, and $4 \%$ progressive disease). Platinum-based chemotherapy is an acceptable alternative for NEC treatment, with a reported response rate of 42 $67 \%$ and a response duration of $8-9$ months $(4,5)$; however, salvage chemotherapy generally exhibits limited treatment effects. The anti-CTLA-4 and anti-PD-1 checkpoint inhibitors for immunotherapy considerably prolong survival in patients with solid tumors, including lung cancer, kidney cancer, and malignant melanoma. For NETs, only NEC of the skin (Merkel cell carcinoma) responds to immunotherapy. Moreover, data in the literature regarding response to immunotherapy remain scant, with marginal 
benefits observed in patients with NETs located elsewhere; the best response (i.e., stable disease) was observed for a patient with pancreatic NEC (6).

The incidence of brain metastases from NETs is approximately $5 \%$, and radiotherapy is essential in treating metastatic disease (7). Both radiotherapy and immunotherapeutic agents have therapeutic effects against brain metastases. Furthermore, because ionizing radiation induces local inflammatory effects, the response to checkpoint blockade immunotherapy may be improved if it is administered in conjunction with radiotherapy. A study demonstrated synergistic effects between radiotherapy and immunotherapy in the treatment of melanoma brain metastases (8).

LM, exhibiting a different anatomy from that of brain metastases, rarely occur in patients with NEC. Although identification of cancer cells in CSF is considered the standard diagnostic test, this invasive procedure still has a high false negative rate. Nevertheless, with improved visualization of the subarachnoid space and improved sensitivity of high-field MRI, this procedure is currently used routinely for diagnosis (9). In terms of the welldocumented dismal outcome of LM, a study reported that whole-brain radiotherapy concurrent with immunotherapy resulted in a favorable response in patients with melanoma LM, although the study was limited by its limited case number (10). A recent retrospective study (11) evaluated patients with melanoma and brain metastases who received radiotherapy, immunotherapy, or both after resection. Patients who received both immunotherapy and radiotherapy had superior survival rates than did those who received radiotherapy alone; moreover, patients who received radiotherapy followed by immunotherapy exhibited superior survival rates than did those who received immunotherapy followed by radiotherapy. However, previous studies have not reported LM from NEC or treatment recommendations. Our case study demonstrated a complete radiological and clinical remission when combined therapies were adopted, accompanied by minimal side effects compared with those of conventional intrathecal or high-dose chemotherapy.

Despite the powerful synergistic effects observed for combined treatment involving immunotherapy and radiotherapy, our patient's primary hepatic tumors had progressed during immunotherapy, which ultimately contributed to her death. The abscopal effect, first mentioned in1953, describes radiotherapy-induced immunemediated tumor regression at sites out of the radiation field.
This effect has been reported in melanoma, kidney cancer, breast cancer, and other metastatic disease. However, the abscopal effect is rare, and only 46 case reports have documented this phenomenon between 1969 and 2014 (12). Questions remain regarding the rate of the abscopal effect and optimization of the abscopal response, including sequence of combined therapies, immunotherapeutic agents, radiation dose, and fraction size for different situations and cancer types. The blood-brain barrier may also limit immunological effects beyond the brain. Considering the lack of reliable data regarding the immunology and advantages of combined therapy in treating NEC, further research is warranted to validate our findings.

In summary, few reports have focused on the treatment of NEC with both immunotherapy and combination of immunotherapy with radiotherapy. Nevertheless, our experience revealed a favorable response of a patient with primary hepatic NEC with leptomeningeal seeding to combined treatment involving immunotherapy and radiotherapy. Checkpoint blockade immunotherapy has evolved and revolutionized current cancer treatment strategies. However, it is not always effective because each cancer has its distinct biology. On the basis of our experience, we recommend that additional studies further elucidate the possible clinical benefits and risks of combined treatment involving radiotherapy and immunotherapy.

\section{Acknowledgments}

Funding: None.

\section{Footnote}

Reporting Checklist: The authors have completed the CARE reporting checklist. Available at http://dx.doi.org/10.21037/ tro-19-103

Conflicts of Interest: All authors have completed the ICMJE uniform disclosure form (available at http://dx.doi. org/10.21037/tro-19-103). JFC serves as an unpaid editorial board member of Therapeutic Radiology and Oncology. The other authors have no conflicts of interest to declare.

Ethical Statement: The authors are accountable for all aspects of the work in ensuring that questions related to the accuracy or integrity of any part of the work are appropriately investigated and resolved. All procedures performed in studies involving human participants were in 
accordance with the ethical standards of the institutional and/or national research committee(s) and with the Helsinki Declaration (as revised in 2013). The consent was waived for this study by the IRB committee at the Taipei Medical University Hospital since the patient died. The study was approved by Taipei Medical University-Joint Institutional Review Board (N201710026).

Open Access Statement: This is an Open Access article distributed in accordance with the Creative Commons Attribution-NonCommercial-NoDerivs 4.0 International License (CC BY-NC-ND 4.0), which permits the noncommercial replication and distribution of the article with the strict proviso that no changes or edits are made and the original work is properly cited (including links to both the formal publication through the relevant DOI and the license). See: https://creativecommons.org/licenses/by-nc-nd/4.0/.

\section{References}

1. Dasari A, Mehta K, Byers LA, et al. Comparative study of lung and extrapulmonary poorly differentiated neuroendocrine carcinomas: A SEER database analysis of 162,983 cases. Cancer 2018;124:807-15.

2. Hallet J, Law CH, Cukier M, et al. Exploring the rising incidence of neuroendocrine tumors: a population-based analysis of epidemiology, metastatic presentation, and outcomes. Cancer 2015;121:589-97.

3. Contessa JN, Griffith KA, Wolff E, et al. Radiotherapy for pancreatic neuroendocrine tumors. Int J Radiat Oncol Biol Phys 2009;75:1196-200.

4. Moertel CG, Kvols LK, O'Connell MJ, et al. Treatment

doi: $10.21037 /$ tro-19-103

Cite this article as: Wang WJ, Lee KD, Lu LS, Ting LL, Chiou JF. Treating leptomeningeal metastases from primary hepatic neuroendocrine carcinoma with combined radiotherapy and immunotherapy: a case report. Ther Radiol Oncol 2021;5:5. of neuroendocrine carcinomas with combined etoposide and cisplatin. Evidence of major therapeutic activity in the anaplastic variants of these neoplasms. Cancer 1991;68:227-32.

5. Mitry E, Baudin E, Ducreux M, et al. Treatment of poorly differentiated neuroendocrine tumours with etoposide and cisplatin. Br J Cancer 1999;81:1351-5.

6. Patnaik A, Kang SP, Rasco D, et al. Phase I study of pembrolizumab (MK-3475; anti-PD-1 monoclonal antibody) in patients with advanced solid tumors. Clin Cancer Res 2015;21:4286-93.

7. Pavel M, Grossman A, Arnold R, et al. ENETS consensus guidelines for the management of brain, cardiac and ovarian metastases from neuroendocrine tumors. Neuroendocrinology 2010;91:326-32.

8. Qian JM, Yu JB, Kluger HM, et al. Timing and type of immune checkpoint therapy affect the early radiographic response of melanoma brain metastases to stereotactic radiosurgery Cancer. 2016;122:3051-8.

9. Pan Z, Yang G, He H, et al. Leptomeningeal metastasis from solid tumors: clinical features and its diagnostic implication. Sci Rep 2018;8:10445.

10. Geukes Foppen MH, Brandsma D, Blank CU, et al. Targeted treatment and immunotherapy in leptomeningeal metastases from melanoma. Ann Oncol 2016;27:1138-42.

11. Pomeranz Krummel DA, Nasti TH, Izar B, et al. Impact of Sequencing Radiation Therapy and Immune Checkpoint Inhibitors in the Treatment of Melanoma Brain Metastases. Int J Radiat Oncol Biol Phys 2020;108:157-63.

12. Abuodeh Y, Venkat P, Kim S. Systematic review of case reports on the abscopal effect. Curr Probl Cancer 2016.40:25-37. 